ThE ASTROPHYSICAL JOURNAL, 375:L45-L48, 1991 July 10

C) 1991. The American Astronomical Society. All rights reserved. Printed in U.S.A.

\title{
THE CHARGE STATE OF THE ANOMALOUS COMPONENT OF COSMIC RAYS
}

\author{
J. H. Adams, Jr., ${ }^{1}$ M. Garcia-Munoz, ${ }^{2}$ N. L. Grigorov, ${ }^{3}$ B. Klecker, ${ }^{4}$ M. A. Kondratyeva, ${ }^{3}$ \\ G. M. Mason, ${ }^{5}$ R. E. McGuire, ${ }^{6}$ R. A. Mewaldt, ${ }^{7}$ M. I. Panasyuk, ${ }^{3}$ \\ Ch. A. Tretyakova, ${ }^{3}$ A. J. Tylka, ${ }^{8}$ and D. A. Zhuravlev ${ }^{3}$ \\ Received 1991 March 4; accepted 1991 April 29
}

\begin{abstract}
The ionic charge state of anomalous cosmic-ray oxygen has been determined by comparing measurements obtained inside the magnetosphere on a series of Cosmos satellite flights with simultaneous observations outside the magnetosphere from IMP 8 and ICE. We find a mean charge state of $0.9_{-0.2}^{+0.3}$ for $\sim 10 \mathrm{MeV}$ nucleon $^{-1}$ anomalous oxygen, consistent with the model of Fisk, Kozlovsky, \& Ramaty, in which the anomalous cosmic rays originate from the neutral component of the local interstellar medium. This same approach gives results consistent with a mean charge state of +7 for solar energetic oxygen ions.

Subject headings: cosmic rays: abundances - cosmic rays: general
\end{abstract}

\section{INTRODUCTION}

Following the discovery of anomalous enhancements in the low-energy spectra of cosmic-ray helium, nitrogen, and oxygen during the 1970s, Fisk, Kozlovsky, \& Ramaty (1974) proposed a theory for the origin of the so-called "anomalous component" (AC) of cosmic rays. In this model the AC comprises interstellar neutral atoms that have been swept into the heliosphere, ionized by the solar wind or solar UV, and then accelerated to energies of $\sim 10 \mathrm{MeV}$ nucleon $^{-1}$ and greater, probably at the solar wind termination shock (Pesses, Jokipii, \& Eichler 1981). For a review of the AC, see Webber (1989) and McKibben (1987). Although the presently available evidence on composition (e.g., Cummings \& Stone 1990) favors this model, Fisk (1979) has pointed out that the clear test is to measure the charge state $(Q)$ of the $\mathrm{AC}$, which is predicted to be $Q=+1$.

There have been numerous attempts to determine $Q$ from studies of the response of the AC to solar modulation. While these investigations tended to show that the AC is singly ionized, they did not always give consistent results. They also relied heavily on assumptions about the nature of the solar modulation process (Cummings, Stone, \& Webber 1984; McDonald et al. 1988; Garcia-Munoz, Pyle, \& Simpson 1990 and references therein).

A less model-dependent approach is to infer $Q$ from the transmission of the AC through the Earth's magnetic field, based on the difference in rigidity between $Q=+1$ and

\footnotetext{
${ }^{1}$ Code 4154, E. O. Hulburt Center for Space Research, Naval Research Laboratory, Washington, DC 20375-5000.

2 Enrico Fermi Institute, University of Chicago, 933 East 56th Street, Chicago, IL 60637.

${ }^{3}$ Research Institute for Nuclear Physics, Moscow State University, Moscow 119899, USSR.

${ }^{4}$ Max-Planck-Institut für Physik und Astrophysik, Institut für Extraterrestrische Physik, D-8046 Garching bei München, Federal Republic of Germany.

${ }^{5}$ Department of Physics and Astronomy and Institute for Physical Sciences and Technology, University of Maryland, College Park, MD 20742.

6 Code 933, NASA/Goddard Space Flight Center, Greenbelt, MD 20771.

7 220-47 Downs Laboratory, California Institute of Technology, Pasadena, CA 91125.

${ }^{8}$ Universities Space Research Association, Code 4154, Naval Research Laboratory, Washington, DC 20375-5000.
}

$Q=+8$ at the same energy per nucleon. This approach has also shown evidence that $Q=+1$ (Grigorov et al. 1988; Oschlies, Beaujean, \& Enge 1989; Singh et al. 1991; Marenny, Guertzen, \& Nymmik 1990; Adams, Beahm, \& Tylka 1991). However, the interpretation of these experiments has been complicated by trapped heavy ions, low AC fluxes, and the lack of simultaneous measurements outside the magnetosphere.

We have taken this same approach, using a series of quiettime measurements of oxygen differential energy spectra in which the trapped heavy ions have been identified and excluded (Grigorov et al. 1988, 1990). We have compared these with simultaneous measurements made outside the magnetosphere to determine the mean $Q$ (hereafter $\langle Q\rangle$ ).

\section{OBSERVATIONS}

From 1984 to 1988 the average composition and energy spectra of $Z \geq 6$ ions were measured inside the magnetosphere approximately 10 times per year using small cellulose nitrate track detector stacks exposed on $\sim 14$ day Cosmos satellite flights (Grigorov et al. 1988). These three-axis stabilized spacecraft were placed in early circular orbits of $62^{\circ}, 73^{\circ}$, or $82^{\circ}$ inclination, at altitudes from 200 to $400 \mathrm{~km}$.

Most of the measurements outside the magnetosphere come from instruments on the IMP 8 satellite. IMP 8 's orbit keeps it in the interplanetary medium most of the time, with brief passages through the distant magnetotail. The measurements we use here are not affected by these magnetotail passages.

Three separate IMP 8 instruments have provided measurements of energetic nuclei for this study. Quiet-time measurements of oxygen from 5 to $28 \mathrm{MeV}$ nucleon ${ }^{-1}$ were provided by the Caltech Electron/Isotope Spectrometer (Mewaldt et al. 1976), which also provided spectra of 1.4-12.5 $\mathrm{MeV}_{\text {nucleon }}{ }^{-1}$ $\mathrm{H}$ and $\mathrm{He}$ nuclei used to select the quiet-time periods for analysis. The Goddard Space Flight Center Very Low Energy Telescope provided oxygen spectra during the solar energetic particle (SEP) events (see McGuire, von Rosenvinge, \& McDonald 1986 and references therein). The University of Chicago instrument (Garcia-Munoz, Mason, \& Simpson 1977) provided quiet-time spectra of $30-200 \mathrm{MeV}$ nucleon ${ }^{-1}$ helium and oxygen nuclei. In addition, the MPI/UMd Ultra-Low Energy Wide Angle Telescope on the ISEE-3/ICE spacecraft 
(Hovestadt et al. 1978) provided quiet-time spectra from 0.6 to $12 \mathrm{MeV}$ nucleon $^{-1}$ during $1985-1986$.

\section{ANALYSIS AND RESULTS}

To identify time periods suitable for this study, we used the IMP 8 spectra of $1.4-12.5 \mathrm{MeV}$ nucleon ${ }^{-1} \mathrm{H}$ and $\mathrm{He}$ from 1985 to 1988. Periods were characterized as "quiet" if the average flux of 4-12.5 MeV nucleon ${ }^{-1} \mathrm{He}$ was less than 3 times the minimum levels for that year, and if the flux of 7-12.5 MeV protons was less than 10 times minimum levels. These criteria allowed for some solar or interplanetary activity, but the spectra of interplanetary 5-28 MeV nucleon ${ }^{-1}$ oxygen during these selected periods have spectral forms and $\mathrm{C} / \mathrm{O}$ ratios that show they are dominated by the AC. Eighteen "quiet" periods were identified for the study. Three periods were also selected in which the interplanetary fluxes were dominated by large SEP events.

During quiet periods, Grigorov et al. (1990) have shown that the anisotropic distribution of tracks in their Cosmos detectors is consistent with two sources: (1) ions trapped in the Earth's magnetic field and (2) ions coming directly from outside the magnetosphere. Also, Grigorov et al. have shown that ion tracks from these sources can be separated by their angles in the track detector stacks, a conclusion that we confirmed with detailed Monte Carlo simulations. Grigorov et al. have also shown that the intensity of the trapped particles follows the intensity of the $\mathrm{AC}$ as it varies over the solar cycle. This behavior is consistent with trapping of the AC, which is expected if $Q=+1$ (Blake \& Friesen 1977). For the work reported here, the flux of ions coming directly from outside the magnetosphere was measured using only tracks within angular limits chosen about the zenith to contain an isotropic distribution of tracks. These angular limits exclude trapped ions.

Elemental composition measurements could be made in the Cosmos stacks at energies greater than $8 \mathrm{MeV}$ nucleon $^{-1}$. During quiet periods the relative composition is $\mathrm{C} / \mathrm{O}=$ $0.04 \pm 0.02, \mathrm{~N} / \mathrm{O}=0.12 \pm 0.04$, and $(Z>8) / \mathrm{O}=0.35 \pm 0.08$, so that $66 \% \pm 10 \%$ of the $Z \geq 6$ events observed were oxygen. Note that the $\mathrm{C} / \mathrm{O}$ ratio is small compared with that of SEPs $(\mathrm{C} / \mathrm{O} \sim 0.5)$ or Galactic cosmic rays $(\mathrm{GCRs})(\mathrm{C} / \mathrm{O} \sim 1)$. It is also small compared with cosmic-ray albedo from the atmosphere $(C / O>1)$. As a result, these sources can make only a very limited contribution to the measured oxygen fluxes. The observed composition is typical of the AC during the 1972 1978 solar minimum (see, e.g., Gloeckler 1979; Mewaldt, Spalding, \& Stone 1984), and it is in agreement with the $\sim 10$

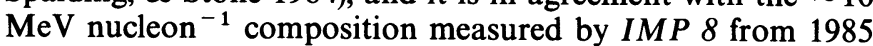
to 1988.

For each of 18 quiet periods the average energy spectrum from Cosmos (4-20 MeV nucleon ${ }^{-1}$ ) was compared with that from IMP 8 (5-28 MeV nucleon ${ }^{-1}$ ) and (during 1985 and 1986 ) from $I C E$, using an orbit-averaged geomagnetic transmission function calculated for each Cosmos flight (Adams, Letaw, \& Smart 1983). Typically, the transmission varies from 0.13 to 0.4 as $Q$ varies from +8 to +1 . For several periods, we verified the calculations with a more thorough treatment which took into account details of the angular acceptance and the actual Cosmos flight path. We also examined the Dst index to make sure that there was no significant geomagnetic activity during the exposures. In addition, we have examined the uncertainties in the geomagnetic cutoff and find that they have a negligible effect on the orbit-averaged transmission.

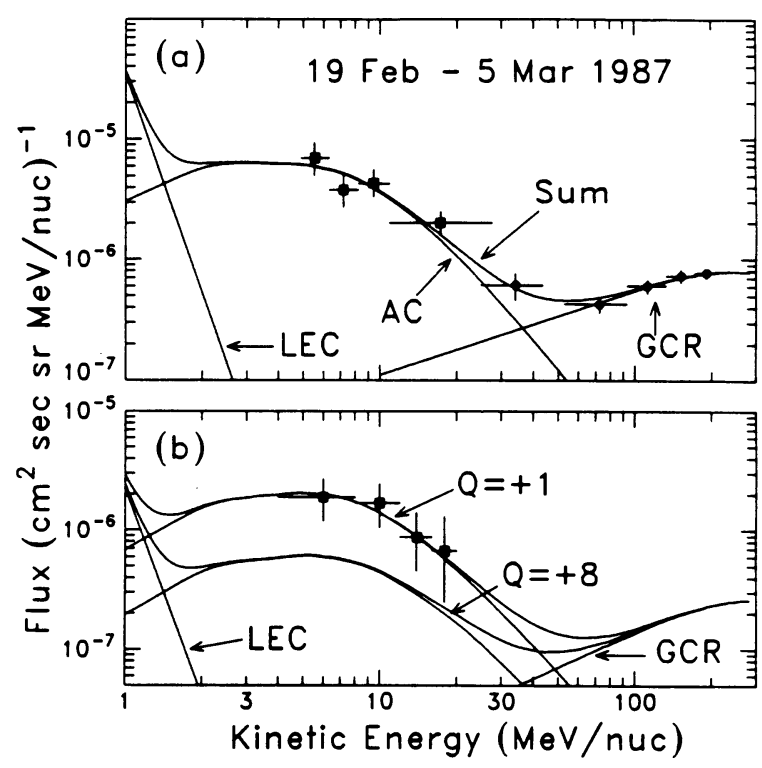

FIG. 1.-Oxygen spectra measured simultaneously inside and outside the magnetosphere during one quiet period near solar minimum. (a) Oxygen spectrum measured outside and deconvolved into solar, anomalous, and Galactic cosmic-ray components. (b) Measured spectrum inside compared with the three components transmitted inside. The transmitted anomalous component is presented for two possible charge states.

The low-energy oxygen flux contains contributions from at least three separate sources which we wish to isolate. These are (1) the GCR component, (2) the AC, and (3) the low-energy component (LEC) of solar (or perhaps interplanetary) origin. The GCR component was determined by fitting GCR spectra from IMP 8 for each of the years from 1985 to 1988 , and extrapolating to lower energies. This component was typically $5 \%-10 \%$ of the observed oxygen flux at $10 \mathrm{MeV}$ nucleon $^{-1}$. The LEC for each period was estimated by fitting a single spectral form to the IMP 8 spectra of $\mathrm{H}$ and He nuclei. The fit to He was scaled by an $\mathrm{O} / \mathrm{He}$ ratio of 0.015 (Cook, Stone, \& Vogt 1984; Mason 1987) to obtain the estimated oxygen LEC. The contribution of this component at $10 \mathrm{MeV}^{\text {nucleon }}{ }^{-1}$ amounted to as much as $10 \%$ in a few cases. A model spectral form for the AC was obtained by fitting the IMP 8 oxygen spectrum summed over all quiet periods from 1985 to 1988 .

These three spectral forms were used to deconvolve the exomagnetospheric oxygen spectra for each period, with only one free parameter, the amplitude of the AC. During the 1986 and 1987 solar minimum period the AC was near its peak intensity at $1 \mathrm{AU}$ and was well measured. Figure $1 a$ shows an example of the deconvolution for one of the periods in 1987. In 1985 and 1988 the AC was less intense, and therefore less well defined.

To estimate the spectra of the three components at Cosmos, they were separately transmitted inside the magnetosphere. We assumed $\langle Q\rangle=+8$ for the GCR component and $\langle Q\rangle=+7$ for the LEC based on the mean charge state of SEP oxygen (Luhn et al. 1984). For AC oxygen we considered both $\langle Q\rangle=+1$ and $\langle Q\rangle+8$. Figure $1 b$ compares simultaneous Cosmos observations with these estimates. Note that $\langle Q\rangle=+1$ is consistent with the data, while $\langle Q\rangle=+8$ is not.

In order to combine data from all 18 periods, we corrected the Cosmos spectra for the estimated GCR and LEC contribu- 


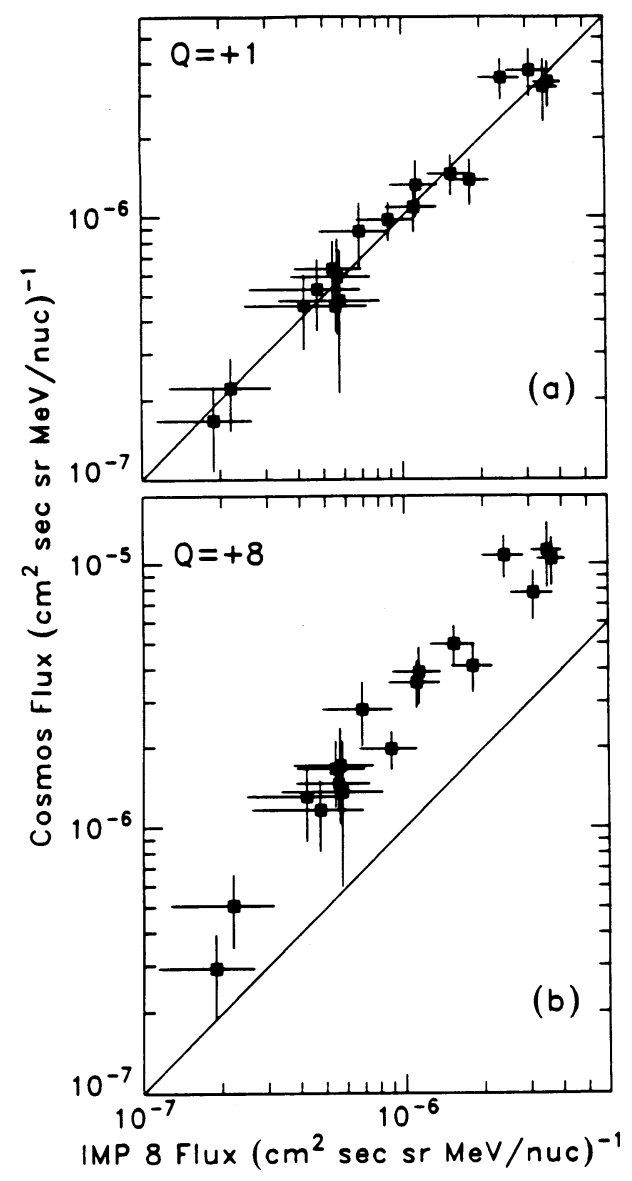

Fig. 2.-Correlation plot of the oxygen flux measurements on IMP 8 and Cosmos. The Cosmos measurements are corrected to outside the magnetosphere assuming charge states of $(a)+1$ and $(b)+8$. The diagonal lines indicate the expected loci of the data points if the flux measurements agree.

tions and then estimated the corresponding AC spectra outside the magnetosphere for both $\langle Q\rangle=+1$ and $\langle Q\rangle=+8$. Figure 2 compares these Cosmos estimates with the IMP 8 measurements, which are also corrected for GCR and LEC contributions. Note that there is good agreement between the two sets of measurements spanning 4 years and a factor of 20 in intensity when $\langle Q\rangle=+1$ is assumed. There are two periods where the statistical uncertainties are too large to distinguish +1 from +8 . The remaining 16 periods are inconsistent with $\langle Q\rangle=+8$. We have found the best-fit $\langle Q\rangle$ for each period. The weighted mean of all 18 periods is $\langle Q\rangle=0.9_{-0.1}^{+0.2}$.

We have considered several possible sources of systematic uncertainty. We believe the dominant sources are uncertainty in the geometry factor and the background corrections for the Caltech instrument on IMP 8 . For these sources we estimate a systematic uncertainty of \pm 0.2 in $\langle Q\rangle$. If we combine this in quadrature with the statistical error, we find $\langle Q\rangle=0.9_{-0.2}^{+0.3}$.

To test our method, we investigated the oxygen charge state during three SEP events. SEP spectra were compared assuming $\langle Q\rangle=+1$ and $\langle Q\rangle=+7$ (Luhn et al. 1984). In all cases the spectra were consistent with $\langle Q\rangle=+7$ (and $\langle Q\rangle=+8$ ) but inconsistent with $\langle Q\rangle=+1$. See, for example, Figure 3, which illustrates the event of 1985 April 25.

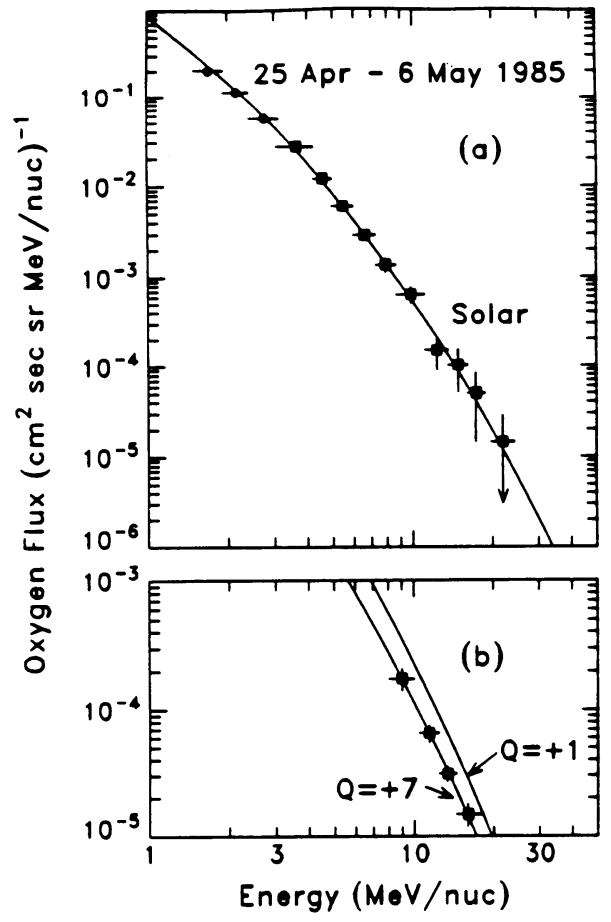

FIG. 3.-Oxygen spectra measured simultaneously inside and outside the magnetosphere during the solar energetic particle event of 1985 April 25. (a) Spectrum measured at $I M P 8$. (b) Spectral fit presented inside for two assumed charge states and compared with Cosmos measurements.

\section{CONCLUSIONS}

We have made a detailed comparison of simultaneous measurements of $\sim 4-20 \mathrm{MeV}$ nucleon $^{-1}$ oxygen inside and outside the magnetosphere from 18 quiet-time periods during 1985-1988. We find that the composition, the energy spectra, and the time variations are all consistent with the conclusion that AC oxygen is the dominant source of particles in both sets of measurements. The differential energy spectra of exomagnetospheric oxygen measured at IMP 8 and Cosmos are consistent if $\langle Q\rangle=+1$, and give a mean value of $0.9_{-0.2}^{+0.3}$. They are generally inconsistent if we assume $\langle Q\rangle=+8$. This same approach shows that SEP oxygen spectra are consistent for $\langle Q\rangle=+7$, as expected, but not for $\langle Q\rangle=+1$.

We conclude that AC oxygen is predominantly singly ionized, as predicted by the theory of Fisk et al. (1974). These measurements, and all other presently available data, are consistent with their assertion that the anomalous cosmic rays originate from the neutral interstellar medium.

We very much appreciate the efforts of NASA and IKI that made possible this joint study under the US/USSR Joint Working Group on Solar Terrestrial Physics. We especially thank Vernon Jones for his participation in our meetings of the US and Soviet teams and his encouragement and support of this work. We also thank D. I. Kozlov, general contractor for Cosmos, for the many flights of track detectors that provided us with the Cosmos data. This work is supported in part by NASA contracts NAG8-678, NAG5-706, W-17,358; NASA grants NAG5-728, NGR 05-002-160, NAGW-1919, NAGW-1990; NSF grant ATM87-20608; and BMFT-FRG contract RV 14B8/74. 
Adams, J. H., Jr., Beahm, L. P., \& Tylka, A. J. 1991, ApJ, in press

Adams, J. H., Jr., Letaw, J. R., \& Smart, D. F. 1983, Cosmic Ray Effects on Microelectronics, Part II, The Geomagnetic Cutoff Effects (NRL Mem. Rept. 5099)

Blake, J. B., \& Friesen, L. M. 1977, Proc. 15th Internat. Cosmic Ray Conf (Plovdiv), ed. C. Ya. Christov, 2, 341

Cook, W. R., Stone, E. C., \& Vogt, R. E. 1984, ApJ, 279, 827

Cummings, A. C., \& Stone, E. C. 1990, Proc. 21 st Internat. Cosmic Ray Conf. (Adelaide), ed. R. J. Protheroe, 6, 202

Cummings, A. C., Stone, E. C., \& Webber, W. R. 1984, ApJ, 287, L99

Fisk, L. A. 1979, in AIP Conf. Proc. 56, Particle Acceleration Mechanisms in Astrophysics, ed. J. Arons, C. McKee, \& C. Max (New York: AIP), 63

Fisk, L. A., Kozlovsky, B., \& Ramaty, R. 1974, ApJ, 190, L35

Garcia-Munoz, M., Mason, G. M., \& Simpson, J. A. 1977, ApJ, 217, 859

Garcia Munoz, M., Pyle, K. R., and Simpson, J. A. 1990, Proc. 21 st Internat. Cosmic Ray Conf. (Adelaide), ed. R. J. Protheroe, 6, 164

Gloeckler, G. 1979, Rev. Geophys. Space Phys., 17, 569

Grigorov, N. L., et al. 1990, Proc. 21st Internat. Cosmic Ray Conf. (Adelaide), ed. R. J. Protheroe, 6, 176

Grigorov, N. L., Bobrovskaya, V. V., Kondratyeva, M. A., Papina, L. P Podgurskaya, A. V., Tretyakova, Ch. A., Zhuravlev, D. A., \& Tretyakova

S. P. 1988, Moscow State University Preprint 88-48/69
REFERENCES

Hovestadt, D., et al. 1978, IEEE Trans., GE-16, 166

Luhn, A., Klecker, B., Hovestadt, D., Gloeckler, G., Ipavich, F. M., Scholer M., Fan, C. Y., \& Fisk, L. A. 1984, Adv. Space Res., 4, 161

Marenny, A. M., Guertzen, G. P., \& Nymmik, R. A. 1990, Nucl. Tracks Rad. Meas., 17, 113

Mason, G. M. 1987, Rev. Geophys., 25, 685

McDonald, F. B., Lal, N., Perko, J. S., von Rosenvinge, T. T., \& Webber, W. R. 1988, ApJ, 333, L109

McGuire, R. E., von Rosenvinge, T. T., \& McDonald, F. B. 1986, ApJ, 301, 938

McKibben, R. B. 1987, Rev. Geophys. Space Phys., 25, 711

Mewaldt, R. A., Spalding, J. D., \& Stone, E. C. 1984, ApJ, 283, 450

Mewaldt, R. A., Stone, E. C., Vidor, S. B., \& Vogt, R. E. 1976, ApJ, 205, 931

Oschlies, K., Beaujean, R., \& Enge, W. 1989, ApJ, 345, 776

Pesses, M. E., Jokipii, J. R., \& Eichler, D. 1981, ApJ, 246, L85

Singh, R. K., Mitra, B., Durgaprasad, N., Biswas, S., Vahia, M. N., Yadav, J. S Dutta, A., \& Goswami, J. N. 1991, ApJ, 374, 753

Webber, W. R. 1989, AIP Conf. Proc. 183, Cosmic Abundances of Matter, ed C. Jake Waddington (New York: AIP), 100 